\title{
Micrometer Observations of Phoebe, the ninth Satellite of Saturn. By E. E. Barnard
}

In the present observations (made with the 40-inch|similar case occurred on December $3 I$, when I had marked refractor) Saturn was $26^{\circ}$ higher on the meridian than when the satellite was observed in 1906 (A.N. 4234). Under these niore favorable conditions Phoebe would necessarily be brightr. I had, nevertheless, expected it to be a faint object. The satellite was sought for on the night of December 8 . After mabing a sketch of the $5 \%{ }^{\prime}$ field, I began measuring the faint stars. It was then noticed that a star marked "I 4 mag.* close north preceding a I $2 \frac{1}{2}$ magnitude star, was changing its position. This proved to be Phoebe. A struments.

fainter star for the satellite.

The transparency of the sky and the seeing were not specially good on any of the nights when the measures were made. It would therefore appear that Phoebe, under fair conditions (at the present opposition of Saturn), must have easily been as bright as the $14^{\text {th }}$ magnitude. Under similar conditions it should be observable with moderate sized inconditions
struments.

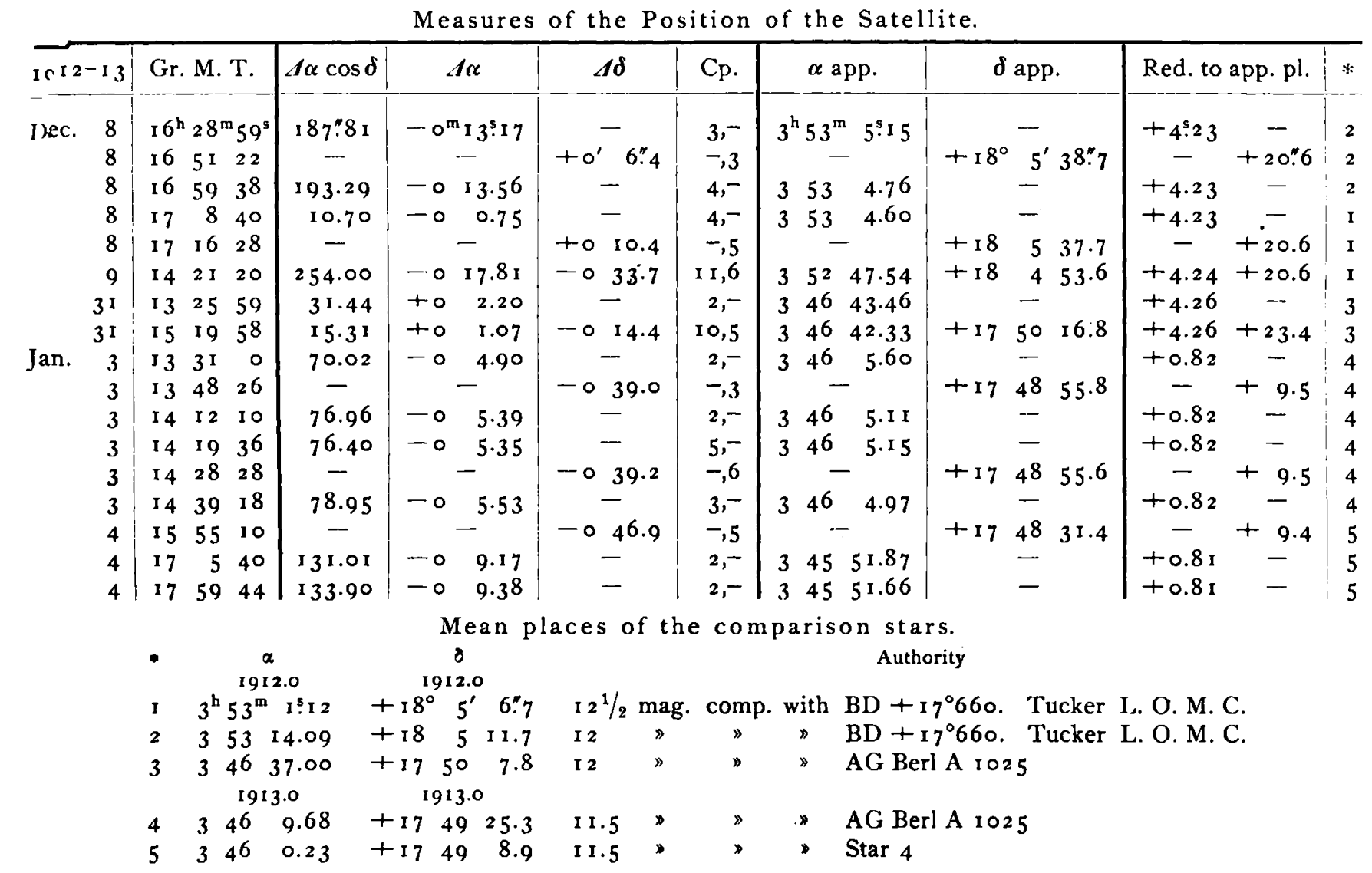

I am greatly indebted to Prof. $R$. H. Tucker who observed the star $\mathrm{BD}+\mathrm{I} 7^{\circ} 660$ with the Lick Observatory M. C. Following is his position: $1912.03^{\mathrm{h}} 53^{\mathrm{m}} 11^{\mathrm{s}} 673+18^{\circ} 3^{\prime} 0^{\prime \prime} 602$ obs. Epoch $1912.97 \mathrm{mag}$. 8.9.

The B. J. Fundamental star places were used for the reduction.

Measures of comparison stars etc.

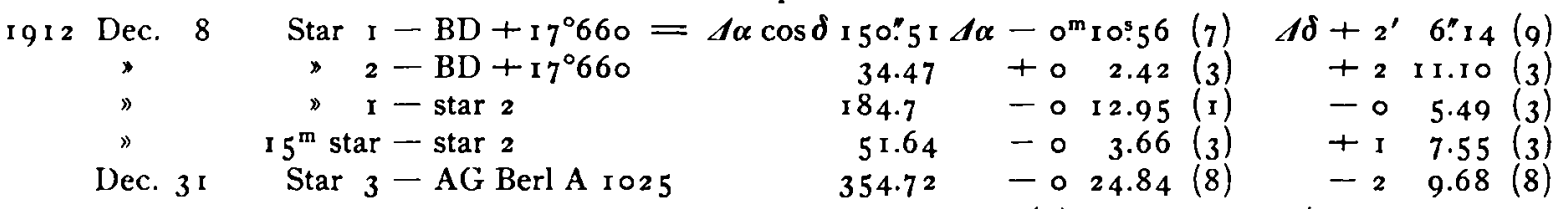

Dec. 3r. A I $^{\mathrm{m}} \cdot 3$ star was compared with star 3: Pos. Ang. 76:58 (5) Dist. $43^{\prime \prime} 65$ (8 double distances).

Position of intermediate star of $1212^{\mathrm{m}}$ used on Dec. $3 \mathrm{I}$ :

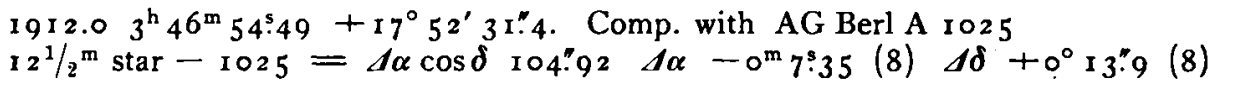

I 9 I Jan. 3 Star $4-A G$ Berl A $1025=\Delta \alpha-0^{m} 55^{s} .59$ (22 tr.) $d \delta-3^{\prime} 3^{\prime \prime} 3$ (5)

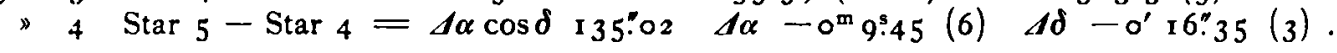


From bad seeing and poor sky on January 3 the satellite was very difficult. On January 4 the sky was very thick and the seeing very bad. Phoebe was seen only once in a while with the greatest difficulty. From this cause the right ascension on that date will not be so accurate as in the previous observations. It was a little better seen at the measures for declination.
The satellite is following almost, if not exactly, the ephemeris of Dr. F. E. Ross given in the American Ephemeris. The corrections on December 8 apparently being

$$
\text { in } \alpha=0.0 \text { in } \delta=0.0 \text {. }
$$

Yerkes Observatory, Williams Bay, Wisconsin, I 9 I 3 Jan. I 8 E. E. Barnara.

\section{Mitteilungen über kleine Planeten.}

Aufnahmen auf der Königstuhl-Sternwarte.

Planet

Position 1913.0

Tägl. Bew.

Gr. Platte

1913 April 28.

190 Ismene $\quad$ I $3^{\mathrm{h}} 16^{\mathrm{m}} .8-4^{\circ} 9^{\prime}-0^{\mathrm{m}} \cdot 5+4^{\prime}$ I $2^{\mathrm{m}} \cdot \mathrm{o}$ A 6940 444 Gyptis

$$
1333 \cdot
$$

\begin{tabular}{|c|c|c|c|c|c|c|c|c|}
\hline 5 Astr & I 4 & 10.8 & - & 348 & -0.8 & +5 & $9 \cdot 5$ & A 694 \\
\hline 42 Isis & 14 & 23.7 & 一 & $\begin{array}{l}249 \\
\end{array}$ & -1.0 & +3 & 10.5 & » \\
\hline $69 \mathrm{H}$ & 14 & 26.5 & - & 73 & -0.7 & +6 & 0.7 & » \\
\hline 9 I 3 & ro & 7.4 & $+I$ & 9 I 2 & +0.2 & -5 & 3.5 & $\begin{array}{l}\mathrm{D} \\
\mathrm{y}\end{array}$ \\
\hline 8 & $I_{4}$ & 39.7 & - & I 36 & -0.8 & 0 & 1.0 & 694 \\
\hline & I 3 & $3^{2.6}$ & - & 9 & -0.7 & +7 & I 1.8 & 319 \\
\hline 36 & I 3 & $3^{6.4}$ & 一 & 49 & -0.8 & +2 & I 2.8 & $n$ \\
\hline 621 & 13 & 0.7 & & I 5 & -0.7 & +3 & I $3 . \mathrm{I}$ & $\searrow$ \\
\hline
\end{tabular}

1913 April 29.

Auf der Platte B 3 r 99 wurden wegen des schlechten Wetters nicht gefunden die Planeten: 2 ro Isabella, 637 [ 1907 YE], 66 Maja und 33 I Etheridgea.

\begin{tabular}{|c|c|c|c|c|c|c|c|c|}
\hline 2 Pallas & I 4 & 48.4 & +23 & 37 & -0.8 & +9 & 8. I & A 695 I \\
\hline & 13 & $5^{8.8}$ & -5 & 54 & -0.5 & +1 & I 5 & $\mathrm{D}_{1074}$ \\
\hline & 12 & 41.0 & + & 549 & -0.7 & +2 & I 2.5 & $\mathrm{~B}_{320 \mathrm{I}}$ \\
\hline & I 2 & 50.6 & + & $\circ 49$ & -0.6 & +5 & I 2.7 & " \\
\hline & I 2 & 54.5 & + & 47 & -0.7 & +2 & I 1.6 & 》) \\
\hline & & & + & & -0.8 & & I 2.0 & \\
\hline
\end{tabular}

1913 April 30.

Platte $\mathrm{m} z \mathrm{~K}$

A $6940 . \quad 9^{\mathrm{h}} 43^{\mathrm{m}} \cdot 5$

$\begin{array}{lll}6944 & 9 & 49.8\end{array}$

$\begin{array}{lll}6948 & \text { I } 3 & 8.2\end{array}$

$695 \mathrm{I} \quad$ I0 9.8

B $3199 \quad 12 \quad 52.2$

$3201 \quad 1043.7$

D 1072958.2

\begin{tabular}{|c|c|c|c|c|}
\hline \multicolumn{4}{|c|}{ Plattenmitte } & Beob. \\
\hline $13^{h}$ & $28^{\mathrm{m}}$ & $-4^{\circ}$ & $40^{\prime}$ & A. Masssinger \\
\hline 14 & 21 & -6 & 15 & $"$ \\
\hline 14 & 44 & $-\mathbf{I}$ & 40 & 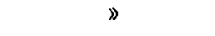 \\
\hline I 4 & 42 & +23 & 30 & $»$ \\
\hline I 3 & 42 & -9 & 10 & F. Kaiser \\
\hline 13 & 5 I & +3 & 50 & $»$ \\
\hline 10 & 7 & +19 & I 2 & M. Wolf \\
\hline I 3 & 59 & -5 & 54 & » \\
\hline
\end{tabular}

1074 I0 14.7

Für Patroclus habe ich durch Anschluß an AG Strb 5006 und 5016 den genauen Ort erhalten: I 9 I 3 April 30 10 $\mathrm{I}^{\mathrm{m}} \cdot 7 \mathrm{~m}$. Z. Kgst.

I913.0: $\alpha=13^{\mathrm{h}} 5^{8^{\mathrm{m}} 46^{\mathrm{s}} .2 \mathrm{I}} \delta=-5^{\circ} 54^{\prime} \mathrm{I}^{\prime \prime} .4$.

Größe nur etwa $15^{\mathrm{m}}$ gegen die Ephemeridenangabe ${ }^{1} 3^{\mathrm{m}}$.

Königstuhl-Sternw., Heidelberg, I 9 I 3 Mai 2.

M. Wolf.

Neuer Planet roi3 RG.

$1913 \mathrm{~m}$. Z. Wien $\alpha$ app. 8 app.

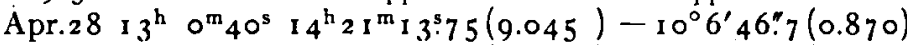

$291042 \quad 4 \quad 1420 \quad 28.96(9.073 n)-1040.3(0.870)$

Größe $13^{\mathrm{m}} \cdot 5$. Tägl. Beweg. $-5^{8.4} \mathrm{I}+2^{\prime} 5^{\prime \prime} .8$.

Wien, k. k. Sternw., I 9 I 3 April 30.

F. Palisa.

Korrektionen von Ephemeriden (B. J. I9I5).

42 Isis I $9{ }_{3}$ Apr. $28+\mathrm{I}^{\mathrm{m}} 9-\mathrm{I}^{\prime}$. Gr.10 ${ }^{\mathrm{m}}$ 5. F.P. Lagrula.

") $z 9+2.0-6$. F. Rheden.

69 Hesperia $》 29-1.2+6$.

240 Vanadis $\gg 29+1.3-7$. Gr. I $2^{m} 5$. F. Palisa.

Ephemeride des Kometen 1912 a (Gale).

$12^{\mathrm{h}} \mathrm{m}$. Z. Berlin. Fortsetzung von A. N. 193.195, $355 ; 194.133$.

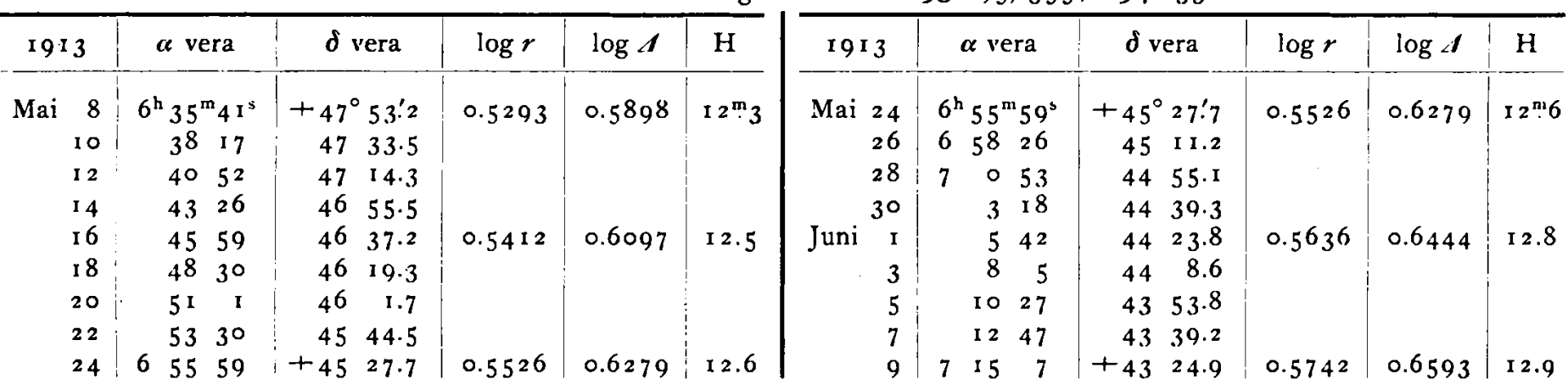

Der Komet ist I9I3 April 26 in Uçcle von Ing. G. Van Biesbroeck und in Bothkamp von Dr. H. H. Kritzinger noch beobachtet worden. Die Ephemeridenkorrektion nach ersterem betrug $-3^{\mathrm{s}}+\mathrm{r} \cdot 5$. Dr. Kritzinger beschreibt den Kometen als elliptischen Nebel von I' und o'.7 Durchmesser, Kernhelligkeit I ${ }^{\prime m} \cdot 8$, Gesamthelligkeit $\mathbf{I}^{\mathrm{m}} \cdot 5$.

Kiel, Bureau der Astronom. Nachr., I 9 I 3 Mai 3.

M. Ebell.

In Algier ward der Komet nach telegraphischer Nachricht von Herrn Direktor $F$. Gonnessiat in Beantwortung einer Anfrage am 2. Mai an der äußersten Grenze der Sichtbarkeit beobachtet, und eine Fortsetzung der Beobachtungen erscheint bei den noch ungunstiger werdenden Sichtbarkeitsverhältnissen auch dort kaum möglich. $K b$.

Inhalt 2u Nr. 4651. E. Gutrrieri. Cometa di Halley igo9 c. 321 . - C. Hoffmeister. Bestimmungen von Meteorbahnen. 327. - E. E. Barnard. Micrometer Observations of Phoebe, the ninth Satellite of Saturn. 335. - Mitteilungen uber kleine Planeten. 335. M. Ebell. Ephemeride des Kometen 1912 a (Gale). 333. 